\title{
Apports des méthodes statistiques et hydrochimiques à la caractérisation des eaux des aquifères fissurés de la région du N'zi-Comoé (Centre-Est de la Côte d'Ivoire)
}

\author{
Michel Amani KOUASSI ${ }^{1 *}$, Alphonse Kouakou YAO ${ }^{1}$, Ernest Kouassi AHOUSSI ${ }^{2}$, \\ Cyrus Laurent SEKI ${ }^{3}$, Anicet N'da YAO $^{3}$, Ismaël Kra KOUASSI ${ }^{3}$ et Jean BIEMI ${ }^{2}$ \\ ${ }^{1}$ Institut National Polytechnique Félix Houphouët Boigny (INP HB), Département des Sciences de la Terre et \\ des Ressources Minières (STeRMi), BP 1093 Yamoussoukro (Côte d'Ivoire). \\ ${ }^{2}$ Université de Cocody, Unité de Formation et de Recherche (UFR) des Sciences de la terre et des Ressources \\ Minières (STRM) ; 22 BP 582 Abidjan 22 ; Tel : (+225) 22483803 (Côte d'Ivoire). \\ ${ }^{3}$ Ecole Supérieure des Mines et de Géologie (ESMG), Institut National Polytechnique Félix Houphouët Boigny \\ (INP HB), BP 1093 Yamoussoukro (Côte d'Ivoire), Tél. (+225) 30646712. \\ *Auteur correspondant, Tél : (+225) 30646715 ; E-mail : michel.a_kouassi@yahoo.fr
}

\section{RESUME}

La région du N'zi-Comoé est située au Centre-Est de la Côte d'Ivoire et appartient du point de vue géologique au socle cristallin. La végétation est constituée de lambeaux de forêt mésophile, de savanes séparées par des forêts-galeries et de jachères, résultat de la déforestation. L'appauvrissement des sols, issu de cette déforestation a eu pour conséquence l'utilisation d'engrais. La déforestation et l'usage des engrais suscitent des interrogations sur la qualité des eaux souterraines du N'zi-Comoé et leur aptitude à la consommation humaine. C'est pour répondre à cette problématique que ce présent travail a été initié. Ainsi, l'objectif de cette étude est la caractérisation hydrogéochimique des eaux et l'analyse de leur aptitude à la consommation humaine. Ce travail a été mené à partir des résultats d'analyses de paramètres physicochimiques des eaux et s'est appuyé sur un échantillon de 193 forages. La méthodologie appliquée est basée sur une approche pluridisciplinaire intégrant l'hydrochimie (diagramme triangulaire de piper, indicateur de temps de séjour) et des analyses statistiques univariées, bivariées et multivariées (ACPN). Du point de vue hydrofaciès, la majorité des eaux des aquifères fissurés du N'zi-Comoé est du faciès bicarbonaté calcique et magnésien. La région du N'zi-Comoé est dominée par deux phénomènes hydrochimiques majeurs : la minéralisation couplée au temps de séjour et l'oxydo-réduction qui sont à l'origine de l'acquisition de la minéralisation des eaux souterraines du N'zi-Comoé. L'étude de la potabilité révèle que les eaux souterraines sont physico-chimiquement conformes dans l'ensemble aux normes recommandées par l'OMS et ne présentent pas de danger majeur pour la consommation humaine.

(C) 2010 International Formulae Group. All rights reserved.

Mots clés: Analyse pluridisciplinaire, faciès hydrochimiques, minéralisation, potabilité, N'zi-Comoé, Côte d'Ivoire.

\section{INTRODUCTION}

La Côte d'Ivoire dispose d'importantes ressources en eau, mais celles-ci sont inégalement réparties dans l'espace et dans le temps. L'eau en Côte d'Ivoire, comme partout ailleurs dans le monde, est source de vie, de 
développement et de santé. C'est pourquoi depuis son accession à l'indépendance, les différents gouvernements qui se sont succédés ont consenti des efforts en matière d'alimentation en eau potable des populations en général, et en particulier les populations rural. Dans ce contexte plusieurs ouvrages hydrauliques ont été réalisés. Selon Anonyme (2009), 20539 ouvrages hydrauliques ont été réalisés jusqu'en 2008 dont 20346 ouvrages d'hydraulique villageoise et 193 ouvrages d'hydraulique villageoise améliorée (HVA). C'est dans cet esprit d'amélioration des conditions de vie en milieu rural que des forages ont été réalisés dans la région du N'ziComoé, ancienne boucle du cacao, à la faveur du projet «Don japonais » au cours de l'année 2000. Aussi, la Côte d'Ivoire indépendante depuis 1960, a-t-elle choisi comme axe majeur pour son développement économique et social la production agricole. Cette politique a eu pour conséquences une destruction abusive de la forêt au profit des plantations. En effet, de 1960 à 2000, la superficie totale des zones forestières non exploitées est passée de 12 millions d'hectares à moins de 2 millions d'hectares au profit des cultures et jachères (BNETD/CCT, 2002 in Brou, 2005). En dehors des rares lambeaux de forêts classées, il n'existe aucun secteur qui n'ait été touché par l'activité humaine, y compris le domaine forestier permanent de l'Etat. Aujourd'hui, l'essentiel du paysage est composé de jachères d'âges différents, de cultures et d'îlots forestiers. L'appauvrissement des sols, issu de la déforestation dans la région du N'zi-Comoé va donc favoriser l'utilisation d'engrais. La déforestation et l'usage des engrais suscitent des interrogations sur la qualité des eaux souterraines du N'zi-Comoé et leur aptitude à la consommation humaine. C'est pour répondre à cette problématique que ce présent travail a été initié. En effet, la qualité des eaux souterraines a toujours suscité un intérêt particulier en raison de son utilisation dans les domaines agricole, industriel mais surtout domestique (El Bouqdaoui et al., 2009). La composition chimique de ces eaux est majoritairement acquise lors de la traversée du sol et de son séjour dans le réservoir. L'eau, au contact de l'encaissant, acquiert une charge minérale caractéristique des roches traversées. Les éléments qui s'y trouvent en solution sont informatifs sur la nature de l'aquifère traversé (Chemseddine et al., 2009; Matini et al., 2009). Cependant, cette qualité peut être altérée et avoir des conséquences dangereuses sur la santé humaine. C'est le cas des polluants azotés (nitrates, nitrites) et des métaux (fer, manganèse) dont l'accroissement des teneurs dans les ressources en eau devient de plus en plus un problème pour l'approvisionnement en eau potable (Alami et al., 2007). Le nitrate, forme plus stable des produits de l'oxydation de l'azote, peut être transformé en nitrite. Les concentrations de nitrite dans la plupart des réserves d'eau sont très faibles (inférieures à $0,1 \mathrm{mg} / \mathrm{l}$ ) (OMS, 2006).

L'objectif de cette étude est la caractérisation hydrogéochimique des eaux des aquifères fissurés de la région du N'ziComoé et l'analyse de leur qualité à la consommation humaine. Le travail a conduit d'abord à identifier les faciès chimiques des eaux souterraines à partir du diagramme de Piper; ensuite, à déterminer les facteurs d'acquisition de la minéralisation des eaux à partir de l'analyse en composantes principales normées (ACPN); enfin, à analyser la potabilité des eaux à la consommation humaine. Dans ce cas, une analyse comparative des caractéristiques statistiques des teneurs en éléments chimiques tels que les nitrates, les nitrites, les métaux (le fer et le manganèse) aux normes OMS a été faite, suivie d'une étude corrélationnelle entre les éléments polluants et les indices de temps de séjour dans la tentative de recherche de l'origine de ces ions. 


\section{MATERIEL ET METHODES \\ Présentation de la zone d'étude}

La région du N'Zi-Comoé, située au Centre-Est de la Côte d'Ivoire est comprise entre les longitudes $3^{\circ} 40^{\prime}$ et $4^{\circ} 55^{\prime}$ Ouest et les latitudes $6^{\circ} 20^{\prime}$ et $8^{\circ} 10^{\prime}$ Nord (Figure 1) avec une superficie de $19560 \mathrm{~km}^{2}$. Sa population est estimée à environ 900000 habitants en 2010, soit une densité moyenne de 45 habitants au $\mathrm{km}^{2}$.

Le relief de la région du N'Zi-Comoé est monotone et constitué d'un plateau légèrement incliné de direction Nord-Sud, avec une altitude moyenne supérieure à 150 m. Les collines de la chaîne Baoulé correspondent au faciès volcano-sédimentaire, principalement les roches vertes. La végétation est constituée de lambeaux de forêt mésophile, et de larges mailles de savanes séparées par des forêts-galeries. Il existe à côté de ces végétations naturelles des végétations liées à l'action anthropique (mosaïques forêt-cultures, mosaïques savanescultures, cultures) (Kouassi, 2007). Le régime climatique a été analysé à partir des données pluviométriques (1961-2000) des stations de Dimbokro, Daoukro, M'bahiakro, Bocanda et Bongouanou. Le climat est de type tropical humide avec une alternance de 4 saisons dont deux (2) saisons pluvieuses et deux (2) saisons sèches. Le régime climatique est caractérisé par des précipitations moyennes mensuelles variant entre $11 \mathrm{~mm}$ et $198 \mathrm{~mm}$. La hauteur pluviométrique annuelle est de $1155 \mathrm{~mm}$.

La région du N'zi-Comoé appartient au domaine paléoprotérozoïque encore appelé domaine baoulé-mossi. Du point de vue lithologique, la zone d'étude est recouverte par un ensemble complexe de formations géologiques constituées essentiellement de roches ignées, métamorphiques et volcanosédimentaires (Daouda, 1998) (Figure 2). On distingue les roches éburnéennes et les roches birimiennes. Les roches éburnéennes sont constituées essentiellement de granitoïdes notamment les granites à deux micas, les granites à biotite homogènes et hétérogènes, et les granodiorites. Les formations birimiennes sont essentiellement des métasédiments et des roches vertes (métavulcanites). Les métasédiments sont constitués en majeure partie de grès et de schistes. Les roches vertes sont constituées de métabasalte, métaandésite, métadolérite, métagabbro, amphibolite. Les différentes formations géologiques ont été affectées par plusieurs systèmes d'accidents majeurs (Géomines, 1982a, 1982b ; Daouda, 1998). Ces fractures sont de direction préférentiellement Nord-Sud. Ces nombreuses fractures sont susceptibles de faciliter la circulation de l'eau dans les roches. Ces formations géologiques ont été également touchées par le phénomène d'altération. Ainsi, l'altération sur le substratum schisteux est particulièrement épaisse et argileuse. Elle est en moyenne d'une trentaine de mètre et peut atteindre $60 \mathrm{~m}$ (Géomines, 1982a, 1982b). En effet, les roches plus ou moins riches en minéraux ferromagnésiens, en minéraux calciques, en plagioclases, en silice et en oxydes ont une altération qui conduit à la formation d'argiles (Yoboué et al., 2010). L'altération des granites est essentiellement sableuse et peu épaisse (inférieure à $10 \mathrm{~m}$ ) (Géomines, 1982a, 1982b). Selon Yoboué et al. (2010), en position topographique de sommet, le fer et le manganèse sont plus liés aux fractions limoneuses et sableuses des sols; seul l'aluminium fait exception à cette règle et se retrouve très souvent dans les feuillets octaédriques des argiles. Au mi-versant, ces éléments se retrouvent plutôt intégrés à la structure des argiles du sol. La fraction sableuse des sols révèle une prédominance des minéraux du fer et du quartz. Plusieurs études ont montré que des faciès cuirassés existent en Côte d'Ivoire (Boulangé et Delvigne, 1973). Des cuirasses ferrugineuses existent plus particulièrement dans la région du N'ziComoé (Bonvallot et Boulangé, 1970). En effet, la cuirasse est la croûte superficielle épaisse (allant jusqu'à plusieurs mètres) 
fortement durcie par des précipitations de fer et d'aluminium se formant surtout en climat intertropical à saisons sèches bien marquées (Foucault et Raoult, 2000 in Okaingni et al. 2009).

\section{Données de l'étude}

L'étude est basée sur un échantillon de 193 forages d'eau. Les données concernent les résultats d'analyses des paramètres physicochimiques réalisées sur ces forages. Les paramètres physiques concernent la température, le $\mathrm{pH}$, la turbidité et la conductivité électrique. Les paramètres chimiques sélectionnés sont le bicarbonate, le calcium, le magnésium, le sodium, le potassium, le chlore, le fer, le manganèse, le nitrate, le nitrite et le sulfate. Les paramètres physiques ont été mesurés in situ. Les échantillons d'eau destinés aux analyses chimiques ont été recueillis dans des bouteilles en polyéthylène pour être analysés au Laboratoire d'Analyse d'Abengourou (LPA). Ces analyses ont été réalisées à l'aide d'un spectrophotomètre DR 2010 de type $\mathrm{HACH}$. Ces données ont été recueillies lors du projet «Don Japonais » au cours de l'année 2000. Elles sont issues de la consultation des fiches techniques de forage mises à notre disposition par la Direction de l'Hydraulique Humaine (DHH).

\section{Analyse statistique des données physico- chimiques}

Des courbes de régression linéaire simple ainsi qu'un critère numérique (coefficient de corrélation) ont été utilisés pour apprécier la qualité des corrélations effectuées entre différentes variables au cours de ce travail. Par définition, on appelle courbe de régression de $\mathrm{y}$ en $\mathrm{x}$, le lieu des moyennes conditionnelles des y liés par $\mathrm{x}$ (de même pour la régression de $\mathrm{x}$ en y) (Laborde, 2000). La corrélation est jugée satisfaisante dans le cas de notre étude lorsque le coefficient de corrélation calculé est supérieur ou égal à 0,70
(Ahoussi, 2008 ; Bahri et al., 2008 ). Selon Bois et al. (2007), le coefficient de corrélation calculé $\mathrm{R}$ est une estimation de la vraie valeur $\rho$. De ce fait, il est intéressant de tester si $\rho$ est significativement différente d'une valeur $\rho_{0}$ donnée. Il est donc important de tester la significativité de la valeur $(0,70)$ choisie. D'après Bois et al. (2007), la variable transformée de Fischer $\mathrm{Z}$ est distribuée normalement avec une moyenne $\bar{Z}$, un écarttype $\sigma$ et une variable réduite u. Les différentes variables sont définies comme suit :

$$
\begin{aligned}
& Z=\frac{1}{2} \log \left(\frac{1+R}{1-R}\right) \\
& \bar{Z}=\frac{1}{2} \log \left(\frac{1+\rho}{1-\rho}\right) \\
& \sigma=\frac{1}{\sqrt{N-3}} \\
& u=\frac{Z-\bar{Z}}{\sigma}
\end{aligned}
$$

Avec $\mathrm{N}$ la taille de l'échantillon égal à 193, le coefficient de corrélation calculé $\mathrm{R}=0,70$ et un taux de confiance de $95 \%$, la zone d'acceptation de l'hypothèse $\mathrm{H}_{0}$ est $1,96<\mathrm{u}<+1,96$, d'après la table de Student. Plusieurs valeurs de $\rho_{0}$ (allant de 0 à 0,95 ) ont été testées et les résultats sont consignés dans le Tableau 1.

Les différents résultats montrent que pour des valeurs de $\rho_{0}$ allant de 0,50 à 0,83 , les valeurs expérimentales de $\mathrm{u}$ sont comprises dans l'intervalle $[-1,96 ;+1,96]$. Ce qui montre que $\rho$ n'est pas significativement différent des valeurs de $\rho_{0}$ allant de 0,50 à 0,83 . Ces résultas démontrent le caractère satisfaisant du choix d'un coefficient de corrélation calculé supérieur ou égal à 0,70 .

Des études statistiques basées sur l'analyse des paramètres descriptifs de séries de valeurs ont été effectuées pour en tirer des informations utiles. Elles ont concerné les valeurs extrêmes (minimum, maximum), la 
moyenne (valeur centrale), l'écart-type (paramètre de dispersion) et le coefficient de variation $(\mathrm{Cv})$ qui se définit comme le rapport de l'écart-type à la moyenne. Selon la valeur de $\mathrm{Cv}$, trois structures de l'ensemble étudié sont mises en évidence :

- si Cv est inférieur à 0,20, la série est très homogène ;

- si Cv est compris entre 0,20 et 0,25, la série est homogène ;

- si Cv est supérieur à 0,25, la série est hétérogène.

Ainsi une évaluation des caractéristiques descriptives des paramètres physiques et chimiques a été faite. En outre, pour la conductivité et la dureté, des histogrammes de fréquence ont été représentés. La conductivité des eaux a été classée selon le degré de minéralisation comme proposé par Rodier (1996) cité par Zourhi et Carlier (2002). La classification d'une eau par rapport à la dureté donne les regroupements suivants (Ahoussi, 2008) :

- la classe des eaux douces pour une dureté inférieure à 15 degrés français ;

- la classe des eaux dures pour une dureté comprise entre 15 et 35 degrés français ;

- la classe des eaux très dures pour une dureté supérieure à 35 degrés français.

Les différents traitements liés aux caractéristiques descriptives et aux corrélations ont été réalisés à partir du tableur Microsoft Excel.

Des analyses en composantes principales normées (ACPN) des variables physico-chimiques ont été effectuées. Cette méthode a été déjà utilisée avec succès par d'autres auteurs (Soro, 2002 ; Touaïbia et al., 2006 ; Lasm et al., 2008; Soro, 2010 ; Ahoussi et al., 2010). La méthode utilisée dans le cadre de cette étude est basée sur l'interprétation des différents facteurs de même que la matrice de corrélation obtenue à la suite du traitement des données. Deux variables seront considérées comme corrélées lorsque leur coefficient de corrélation est supérieur ou égal à 0,7 (Ahoussi, 2008). L'ACPN a été effectuée sur 193 échantillons d'eau. Les variables physico-chimiques prises en compte pendant cette analyse sont au nombre de 11 ( $\mathrm{pH}$, minéralisation totale (MT), la Conductivité Cond., $\mathrm{Ca}^{2+}, \mathrm{K}^{+}, \mathrm{Na}^{+}, \mathrm{Mg}^{2+}$, $\mathrm{Cl}^{-}, \mathrm{HCO}_{3}^{-}, \mathrm{SO}_{4}^{2-}$ et $\mathrm{N}_{3}^{-}$). Les différents traitements ont été réalisés à partir du logiciel STATISTICA 6.0.

\section{Analyse hydrochimique des données}

La classification hydrogéochimique des eaux souterraines a été réalisée à l'aide du diagramme triangulaire de Piper qui est très fréquemment utilisé dans le domaine de l'hydrochimie avec de très bons résultats (Yermani et al., 2003 ; Mohammad et Hamed, 2004 ; Tabouche et Achour, 2004 ; Alayat et Lamouroux, 2007 ; Kouzana et al., 2007 ; Oga et al., 2009). Ainsi, l'identification des facteurs qui gouvernent la minéralisation des eaux souterraines conduisant à différents faciès chimiques définis par le diagramme triangulaire de Piper a été réalisée à partir de l'analyse en composantes principales normées (ACPN).

La potabilité de l'eau est définie par des paramètres physiques, chimiques et biologiques, mais surtout en fonction de son usage. Dans cette étude, la potabilité de l'eau a été analysée au regard de l'usage humain et a concerné uniquement les paramètres physiques et chimiques. La comparaison des teneurs en éléments physiques et chimiques des eaux des différents forages aux normes de l'Organisation Mondiale de la Santé (OMS, 2006) a été effectuée (Lallahem, 2002). L'analyse a porté particulièrement sur les composés azotés tels que les nitrates et nitrites, et des métaux tels que le fer et le manganèse. Les eaux ont été donc jugées potables, pour un paramètre donné, si les valeurs des paramètres de caractérisation 
définis plus haut sont dans les intervalles recommandés par l'Organisation Mondiale de la Santé (OMS, 2006). Une valeur calculée $\left(\mathrm{V}_{\mathrm{c}}\right)$ a été jugée proche d'une valeur de référence donnée $\left(\mathrm{V}_{\mathrm{r}}\right)$ si l'écart relatif évalué $\left(\mathrm{E}_{\mathrm{r}}\right)$ est inférieur ou égal à $10 \%$. Cet écart relatif (Vr) s'exprime comme suit (équation 5):

$$
E_{r}=\left|\frac{V_{r}-V_{c}}{V_{r}}\right|
$$

Un indicateur qualitatif du temps de séjour des eaux dans les aquifères a été calculé. Il a permis d'apprécier l'âge relatif des eaux. Cet indicateur de temps de séjour I est fonction des concentrations en $\mathrm{Ca}^{2+}, \mathrm{Mg}^{2+}$, $\mathrm{Na}^{+}$et $\mathrm{K}^{+}$et est calculé selon l'équation 6 (Lalbat, 2006):

$I=\frac{M g^{2+} \times\left(\mathrm{Na}^{+}+\mathrm{K}^{+}\right)}{\left(\mathrm{Ca}^{2+}\right)^{2}}$

Cet indice est d'autant plus grand que le temps de séjour de l'eau dans la formation aquifère est long (Lalbat, 2006). Une analyse corrélationnelle basée sur la régression linéaire entre les indices de temps de séjours calculés et les teneurs des composés azotés (nitrates et nitrites) et certains métaux (fer, manganèse), a été réalisée dans le but de rechercher les origines probables de ces éléments polluants.

\section{RESULTATS}

Analyse de la fiabilité et de l'homogénéité des données

Le graphique de corrélation des sommes des concentrations des anions en fonction de celles des cations (Figure 3) met en évidence un coefficient de corrélation d'une valeur de 0,87 (supérieure à 0,70). Ce résultat traduit une bonne corrélation linéaire entre ces deux variables et par conséquent une balance ionique satisfaisante. On peut donc conclure que les différentes données utilisées sont fiables.
Les résultats de l'étude du coefficient de variation des paramètres physicochimiques ont permis d'apprécier l'homogénéité des différentes données (Tableau 2). Les paramètres physiques tels que la température et le $\mathrm{pH}$ sont homogènes $\left(\mathrm{C}_{\mathrm{V}}<0,25\right)$ alors que la turbidité et la conductivité sont hétérogènes $\left(C_{V}>0,25\right)$. Les coefficients de variation des paramètres chimiques sont tous supérieurs ou égaux à $0,43 \quad\left(\mathrm{C}_{\mathrm{V}}>0,25\right)$, ce qui traduit une forte hétérogénéité des différentes valeurs des paramètres chimiques. La dispersion des valeurs du nitrate est très remarquable avec un coefficient de variation égal à 2 soit $200 \%$. Dans l'ensemble, les paramètres physiques sont plus homogènes que les paramètres chimiques.

\section{Analyse des valeurs des paramètres physiques des eaux souterraines de la région du N'zi-Comoé}

D'après le Tableau 1, les eaux souterraines de la région du N'Zi-Comoé ont des températures variant entre $24,4{ }^{\circ} \mathrm{C}$ et 31,6 ${ }^{\circ} \mathrm{C}$ avec une moyenne de $27,18^{\circ} \mathrm{C}$. Le $\mathrm{pH}$ de ces eaux est compris généralement entre 5,12 et 8,21. La majorité des eaux du N'zi-Comoé a un $\mathrm{pH}$ moyen égal à 6,58 , ce qui est proche de 7 . En effet, l'écart relatif entre la référence 7 et la valeur moyenne 6,58 est de $6 \%$ qui est inférieure à $10 \%$. Ces eaux sont donc acceptables pour la boisson. La turbidité des eaux souterraines du N'zi-Comoé reste faible dans l'ensemble. Les valeurs des mesures varient entre 0,33 NTU et 3,49 NTU, avec une moyenne de 0,79 NTU. Elles sont en grande partie inférieures à l'unité tandis que la limite de turbidité fixée par l'OMS (2006) est de 5 NTU. Les eaux de la région contiennent peu de particules en suspension. La conductivité des eaux varie entre 106 et $1535 \mu \mathrm{s} / \mathrm{cm}$. La plus forte valeur est observée dans le village de Soungassi (Dimbokro). En moyenne, la conductivité de ces eaux est de 391,40 $\mu \mathrm{s} / \mathrm{cm}$. Dans la zone d'étude, $69 \%$ des points d'eaux 
étudiés présentent une conductivité comprise entre 250 et $750 \mu \mathrm{s} / \mathrm{cm}$ (Figure 4a). La dureté des eaux varie entre $2,80{ }^{\circ} \mathrm{F}$ et $58,47^{\circ} \mathrm{F}$, avec une moyenne de $13,42{ }^{\circ} \mathrm{F}$. Les eaux de la région sont douces à l'exception de celles de N'Goulé Kouamékro $\left(55,47^{\circ} \mathrm{F}\right.$, Daoukro) et Soungassi $\left(58,47^{\circ} \mathrm{F}\right.$, Dimbokro) qui sont des eaux très dures (Figure 4b).

\section{Analyse hydrogéochimique des eaux} souterraines de la région du N'zi-Comoé

La projection du chimisme des eaux dans le diagramme de Piper a révélé deux faciès: le faciès bicarbonaté calcique et magnésien et le faciès chloruré calcique et magnésien (Figure 4). Les eaux bicarbonatées calciques et magnésiennes sont les plus dominantes de la région du N'Zi-Comoé et sont présentes dans tous les départements. 99\% des points d'eau se rangent dans cette classe. Les espèces ioniques dominantes de ces eaux sont le bicarbonate et le calcium. Les eaux chlorurées calciques et magnésiennes sont très rares dans la région. Elles représentent seulement $1 \%$ des points d'eau. Les localités concernées sont celles de Nofou gare (Dimbokro) et Niandian (Bongouanou). Les espèces ioniques dominantes de ces eaux sont les chlorures et le calcium.

\section{Analyse des facteurs de la minéralisation des eaux souterraines de la région du N'zi- Comoé}

Les résultats de l'ACP sont résumés dans les Tableaux 3 et 4. La liaison existant entre toutes les variables prises deux à deux et les coefficients de corrélation entre ces différentes variables sont donnés par la matrice de corrélation (Tableau 3).

Cette matrice de corrélation montre qu'il existe une bonne corrélation entre, d'une part, la minéralisation totale, et d'autre part, les alcalins $(0,94)$, le $\mathrm{HCO}_{3}{ }^{-}(0,94)$, le $\mathrm{Ca}^{2+}$ $(0,90)$, la dureté totale $(0,89)$ et le $\mathrm{Mg}^{2+}$ $(0,68)$. La conductivité électrique est généralement corrélée aux cations majeurs et anions majeurs en dehors du $\mathrm{SO}_{4}{ }^{2-}$ et $\mathrm{NO}_{3}{ }^{-}$. Elle est fortement liée à la minéralisation totale $(0,90)$ et à la dureté totale $(0,90)$. Les $\mathrm{Ca}^{2+}$ sont corrélés aux alcalins $(0,74)$, aux bicarbonates $(0,74)$ et à la dureté $(0,97)$. Le sodium (Na) est parfaitement corrélé au potassium (1) et aux bicarbonates (1). Il est également corrélé à la dureté $(0,71)$. Le potassium est parfaitement corrélé aux bicarbonates et assez bien corrélé à la dureté $(0,71)$. Les éléments ne présentant apparemment aucune corrélation satisfaisante avec les autres variables sont le fer, le Manganèse et les sulfates.

Le Tableau 4 présente les valeurs propres, les variances exprimées pour chaque facteur et leur cumul.

Le facteur $F 1$, avec une variance exprimée de $49,40 \%$, est le plus important de tous, ensuite viennent les facteurs F2 et F3, avec respectivement 12,70 et $11,92 \%$ de la variance exprimée. Le cumul de la variance exprimée est de $74,02 \%$ pour les trois facteurs. Ces axes factoriels retenus pour cette analyse statistique sont donc représentatifs de la variance de l'ensemble des données. Les plans factoriels F1-F2 et F1-F3 représentent donc respectivement des variances cumulées égales à $62,10 \%$ et à $61,32 \%$. Ces deux plans non seulement représentent presque la même variance cumulée mais également intègrent suffisamment de représentativité pour mettre en évidence les tendances générales. Les différents résultats étant presque les mêmes, seuls ceux du plan F1-F3 ont été présentés parce que ce plan marque plus nettement la distinction des tendances.

L'analyse des résultats de l'ACP de l'espace des variables du plan factoriel F1-F3 montre que le facteur F1, le plus important $(49,40 \%)$, est déterminé par $\mathrm{MT}, \mathrm{Ca}^{2+}, \mathrm{CE}$, $\mathrm{Na}^{+}, \mathrm{K}^{+}, \mathrm{HCO}_{3}{ }^{-}$, THT, $\mathrm{Mg}^{2+}, \mathrm{SO}_{4}{ }^{2-}$ (Figure 6). Le facteur F1 regroupe donc l'essentiel des éléments chimiques et la conductivité électrique. La proximité de ces variables dans le cercle de communauté montre qu'elles 
commandent le phénomène de mise en solution des éléments minéraux. Ces variables mettent en évidence un processus lent de mise en solution des ions. Ainsi, le facteur F1 semble rendre compte des conditions d'acquisition du chimisme de l'eau. Il explique le phénomène de minéralisationtemps de séjour qui est le mécanisme le plus dominant dans le processus d'acquisition d'ions par l'eau en milieu de socle. La plus grande partie des variables est corrélée positivement avec ce phénomène montrant ainsi que le principal phénomène à l'origine de la minéralisation des eaux souterraines de la région est l'hydrolyse. Cette mise en solution est due au contact pendant un temps de séjour suffisant des eaux avec les formations géologiques encaissantes. Le facteur 3 qui exprime 11,92\% de l'inertie du nuage des points représentatifs des ouvrages est déterminé par les éléments : $\mathrm{Mn}^{2+}, \mathrm{Fe}^{2+}$ et Tub (Figure 6). Ces résultats montrent que le facteur 3 n'est pas lié au phénomène d'hydrolyse mais plutôt au phénomène d'oxydo-réduction. Ce phénomène est à l'origine de turbidité des eaux.

La représentation graphique dans l'espace factoriel des unités statistiques (Figure 7) rend compte de la répartition des points d'eau en fonction des différents facteurs F1-F3. L'analyse de ce graphe montre deux principaux regroupements des points d'eau. La classe 1 regroupe les eaux les plus minéralisées et ayant les plus fortes conductivités électriques dont l'acquisition ionique est sous le contrôle de la minéralisation-temps de séjour $(98 \%)$. La classe 2 renferme les eaux dont les minéralisations totales sont faibles. Elles sont surtout caractérisées par des valeurs de turbidité les plus grandes de la région et/ou des teneurs en fer et en manganèse élevées. Cette classe est constituée par quatre échantillons (2\%) que sont les forages de Yobouéssou (Bongouanou), Konandi N'Dakro (M'Bahiakro), Anzan Kouamekro (M'Bahiakro) et de Kouassi kouakoukro (Dimbokro). Ces échantillons présentent également les plus faibles valeurs de dureté totale notamment à Kouassi kouakoukro (8 $\left.{ }^{\circ} \mathrm{F}\right)$ et de faibles valeurs de conductivité électrique (Yobouéssou, $247 \mu \mathrm{S} / \mathrm{cm}$ ).

\section{Analyse des résultats de la potabilité des eaux souterraines de la région du N'zi- Comoé \\ L'analyse de la potabilité à la boisson des eaux souterraines de la région du N'zi- Comoé a concerné les composés azotés (nitrates et nitrites) et certains métaux (fer, manganèse). Les teneurs en nitrates sont acceptables car elles sont toutes en dessous de}

Tableau 1 : Paramètres caractéristiques du test de validation de R.

\begin{tabular}{ccccc}
\hline $\begin{array}{c}\boldsymbol{\rho}_{\mathbf{0}} \text { (valeur vraie } \\
\text { testée) }\end{array}$ & $\begin{array}{c}\mathbf{Z} \text { (variable de } \\
\text { Fischer) }\end{array}$ & $\bar{Z}$ (Moyenne) & $\boldsymbol{\sigma}$ (Ecart-type) & u calculé \\
\hline 0,00 & 0,38 & 0,00 & 0,07 & 5,19 \\
0,50 & 0,38 & 0,24 & 0,07 & 1,90 \\
0,75 & 0,38 & 0,42 & 0,07 & $-0,63$ \\
0,83 & 0,38 & 0,52 & 0,07 & $-1,92$ \\
0,84 & 0,38 & 0,53 & 0,07 & $-2,12$ \\
0,95 & 0,38 & 0,80 & 0,07 & $-5,77$ \\
\hline
\end{tabular}


M. A. KOUASSI et al. / Int. J. Biol. Chem. Sci. 4(5): 1816-1838, 2010

Tableau 2: Caractéristiques statistiques des paramètres physico-chimiques analysés.

\begin{tabular}{|c|c|c|c|c|c|c|}
\hline & & \multicolumn{5}{|c|}{ Paramètres statistiques } \\
\hline & & Min & Max & Moy & Ecart-type & Coefficient de variation $(\mathrm{Cv})$ \\
\hline \multirow{4}{*}{ 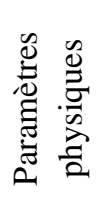 } & Température $\left(\mathrm{T}^{\circ} \mathrm{C}\right)$ & 21,40 & 31,60 & 27,18 & 1,37 & 0,05 \\
\hline & $\mathrm{pH}$ & 5,12 & 8,21 & 6,58 & 0,48 & 0,07 \\
\hline & Turbidité (NTU) & 0,33 & 3,49 & 0,79 & 0,37 & 0,47 \\
\hline & conductivité $(\mu \mathrm{S} / \mathrm{cm})$ & 106 & 1535,00 & 391,40 & 200,53 & 0,51 \\
\hline \multirow{9}{*}{ 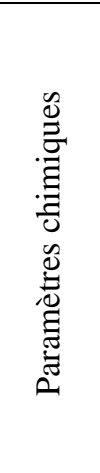 } & $\mathrm{Ca}^{2+}(\mathrm{mg} / \mathrm{l})$ & 4,01 & 155,00 & 32,51 & 23,66 & 0,73 \\
\hline & $\mathrm{Mg}^{2+}(\mathrm{mg} / \mathrm{l})$ & 1,94 & 48,60 & 13,02 & 6,83 & 0,52 \\
\hline & $\mathrm{Na}^{+}$ & 0,95 & 7,06 & 3,27 & 1,40 & 0,43 \\
\hline & $\mathrm{K}^{+}$ & 0,42 & 3,14 & 1,46 & 0,62 & 0,43 \\
\hline & $\mathrm{HCO}_{3}^{-}(\mathrm{mg} / \mathrm{l})$ & 61 & 451,40 & 209,05 & 89,64 & 0,43 \\
\hline & $\mathrm{Cl}^{-}(\mathrm{mg} / \mathrm{l})$ & 3,55 & 92,20 & 17,61 & 13,31 & 0,76 \\
\hline & $\mathrm{NO}_{3}^{-}(\mathrm{mg} / \mathrm{l})$ & 0 & 27,00 & 1,5 & 3,02 & 2,00 \\
\hline & $\mathrm{SO}_{4}^{2-}(\mathrm{mg} / \mathrm{l})$ & 0 & 26,00 & 5,09 & 4,92 & 0,97 \\
\hline & TH total $\left({ }^{\circ} \mathrm{F}\right)$ & 2,80 & 58,48 & 13,42 & 8,05 & 0,60 \\
\hline
\end{tabular}


M. A. KOUASSI et al. / Int. J. Biol. Chem. Sci. 4(5): 1816-1838, 2010

Tableau 3 : Matrice de corrélation entre les variables étudiées.

\begin{tabular}{|c|c|c|c|c|c|c|c|c|c|c|c|c|c|c|}
\hline & Tub & $\mathrm{CE}$ & MT & pH & $\mathrm{Ca}^{2+}$ & $\mathrm{Mg}^{2+}$ & $\mathrm{Na}^{+}$ & $\mathbf{K}^{+}$ & $\mathrm{Fe}^{2+}$ & $\mathbf{M n}^{2+}$ & $\mathrm{HCO}_{3}^{-}$ & $\mathrm{Cl}^{-}$ & $\mathrm{SO}_{4}^{-}$ & $\mathrm{NO}_{3}$ \\
\hline Tub & 1,00 & & & & & & & & & & & & & \\
\hline $\mathbf{C E}$ & $-0,17$ & 1,00 & & & & & & & & & & & & \\
\hline MT & $-0,14$ & 0,90 & 1,00 & & & & & & & & & & & \\
\hline pH & $-0,06$ & 0,27 & 0,38 & 1,00 & & & & & & & & & & \\
\hline $\mathrm{Ca}^{2+}$ & $-0,16$ & $\mathbf{0 , 8 8}$ & $\mathbf{0 , 8 9}$ & 0,27 & 1,00 & & & & & & & & & \\
\hline $\mathrm{Mg}^{2+}$ & $-0,02$ & 0,75 & 0,68 & 0,10 & 0,68 & 1,00 & & & & & & & & \\
\hline $\mathrm{Na}^{+}$ & $-0,12$ & 0,74 & 0,94 & 0,45 & 0,74 & 0,49 & 1,00 & & & & & & & \\
\hline $\mathbf{K}^{+}$ & $-0,12$ & 0,74 & 0,94 & 0,45 & 0,74 & 0,49 & 1,00 & 1,00 & & & & & & \\
\hline $\mathrm{Fe}^{2+}$ & 0,63 & $-0,16$ & $-0,13$ & $-0,05$ & $-0,14$ & $-0,02$ & $-0,12$ & $-0,12$ & 1,00 & & & & & \\
\hline $\mathrm{Mn}^{2+}$ & 0,29 & $-0,12$ & $-0,10$ & $-0,04$ & $-0,12$ & 0,02 & $-0,09$ & $-0,09$ & 0,65 & 1,00 & & & & \\
\hline $\mathrm{HCO}_{3}^{-}$ & $-0,12$ & 0,74 & 0,94 & 0,45 & 0,74 & 0,49 & $\mathbf{1 , 0 0}$ & $\mathbf{1 , 0 0}$ & $-0,12$ & $-0,09$ & 1,00 & & & \\
\hline $\mathrm{Cl}^{-}$ & $-0,12$ & 0,71 & 0,50 & $-0,03$ & 0,59 & 0,62 & 0,19 & 0,19 & $-0,09$ & $-0,09$ & 0,19 & 1,00 & & \\
\hline $\mathrm{SO}_{4}^{-}$ & $-0,04$ & 0,66 & 0,70 & 0,27 & 0,54 & 0,47 & 0,64 & 0,64 & $-0,10$ & $-0,09$ & 0,64 & 0,35 & 1,00 & \\
\hline $\mathrm{NO}_{3}^{-}$ & $-0,01$ & 0,56 & 0,44 & 0,07 & 0,46 & 0,46 & 0,27 & 0,27 & $-0,06$ & $-0,04$ & 0,27 & 0,46 & 0,49 & 1,00 \\
\hline
\end{tabular}


Tableau 4 : Valeurs propres et pourcentages exprimés pour les axes principaux.

\begin{tabular}{lccc}
\hline & F1 & F2 & F3 \\
\hline Valeurs propres & 8,4 & 2,16 & 2,03 \\
\% Total de la variance exprimée & 49,4 & 12,7 & 11,9 \\
Cumul de valeurs propres & 8,4 & 10,6 & 12,6 \\
Cumul de la variance exprimée (\%) & 49,4 & 62,1 & 74 \\
\hline
\end{tabular}

Tableau 5 : Caractéristiques statistiques des valeurs des indices de temps de séjour.

\begin{tabular}{ll}
\hline Minimum & 0,009 \\
\hline Maximum & 1,763 \\
Moyenne & 0,158 \\
Ecart-type & 0,211 \\
Coefficient de variation & 1,339 \\
\hline
\end{tabular}

la limite fixée par l'OMS (50 mg/l). Cependant, 5 points d'eau, soit $2,6 \%$, présentent des valeurs supérieures à $10 \mathrm{mg} / \mathrm{l}$ et les valeurs fluctuent entre 13 et $35,8 \mathrm{mg} / \mathrm{l}$. Du point de vue des nitrates, les eaux de la région sont donc aptes à la consommation humaine. Les nitrites sont généralement absents dans ces eaux souterraines. Cependant, des teneurs non nulles mais relativement faibles et inférieures à la norme OMS $\left(0,1 \mathrm{mg} \cdot \mathrm{L}^{-1}\right)$ ont été obtenues dans le Sud de la zone d'étude au niveau de Bongouanou. Les teneurs en fer $\left(\mathrm{Fe}^{2+}\right)$ varient de 0 à $2,5 \mathrm{mg} / \mathrm{l}$ avec une moyenne de $0,19 \mathrm{mg} / \mathrm{l}$. Cependant, $15 \%$ des échantillons correspondant à 29 points d'eau ont des teneurs en fer au-delà de la norme recommandée par l'OMS (2006) qui est de 0,3 $\mathrm{mg} / \mathrm{l}$. Ces points sont dispersés sur toute la zone d'étude. En ce qui concerne les teneurs en manganèse, elles varient de 0 à $0,9 \mathrm{mg} / \mathrm{l}$ avec une moyenne de $0,04 \mathrm{mg} / \mathrm{l}$. Seulement $9 \%$ des valeurs sont au-delà de $0,1 \mathrm{mg} / \mathrm{l}$, norme recommandée par l'OMS (2006).
Les valeurs des indices de temps de séjour sont consignées dans le Tableau 5. Ces valeurs sont comprises entre 0,009 et 1,763 avec une moyenne de 0,158 et un écart-type de 0,211 . Le coefficient de variation, d'une valeur de 1,339 indique que les indices de temps de séjour sont très dispersés donc très hétérogènes.

Dans une tentative de recherche de l'origine des polluants étudiés, des corrélations entre les teneurs des polluants et les indices de temps de séjour ont été réalisées. Les coefficients de corrélation obtenus sont respectivement de 0 pour les nitrates (Figure 8a), 0,03 pour les nitrites (Figure 8b), 0,27 pour le fer (Figure 8c) et 0,25 pour le manganèse (Figure $8 \mathrm{~d}$ ). Les faibles valeurs des coefficients de corrélation montrent que ces différents ions ne semblent pas issus du processus de minéralisationtemps de séjour. 


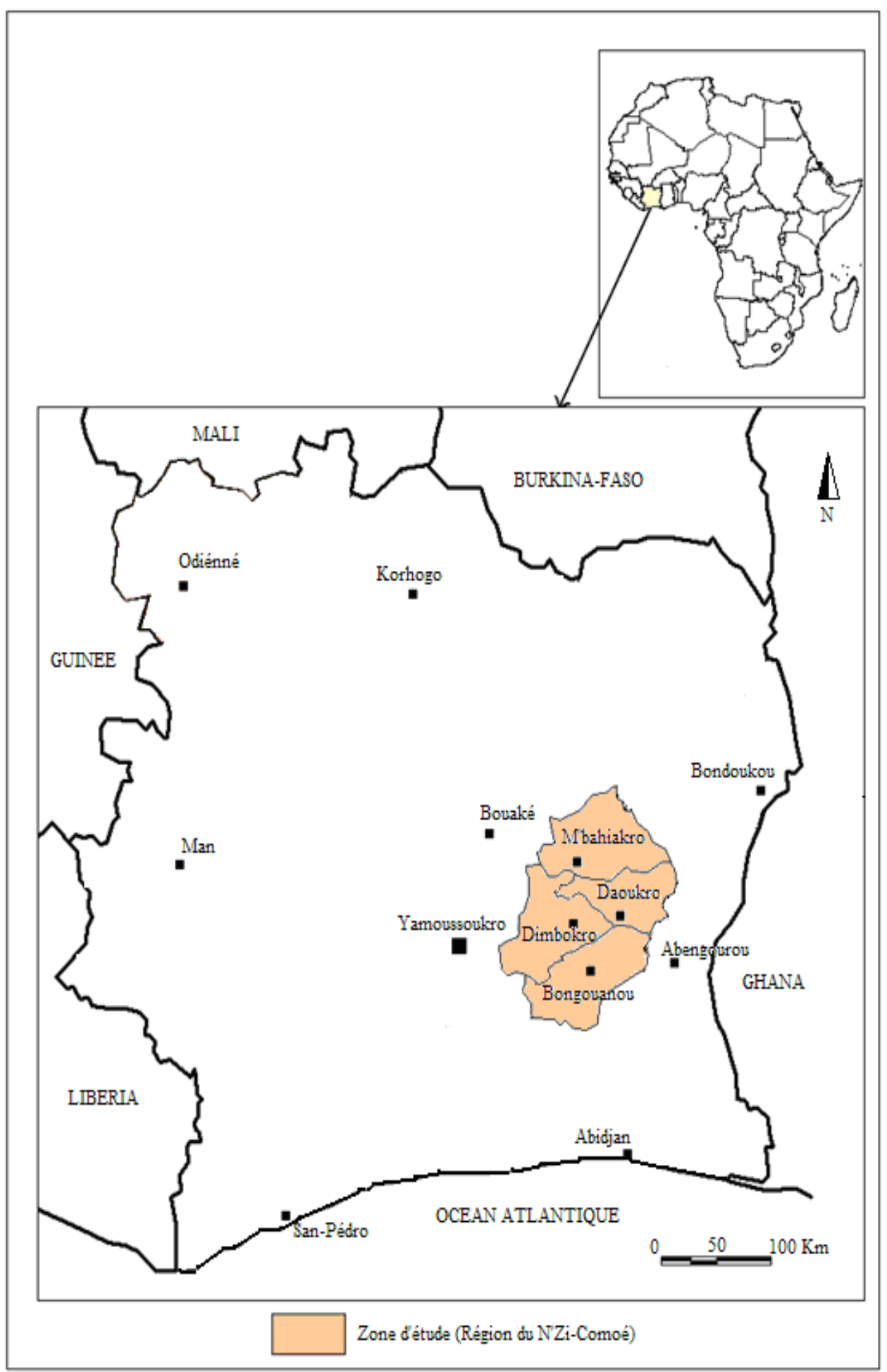

Figure 1 : Présentation de la région du N'Zi-Comoé (Centre-Est de la Côte d'Ivoire) 


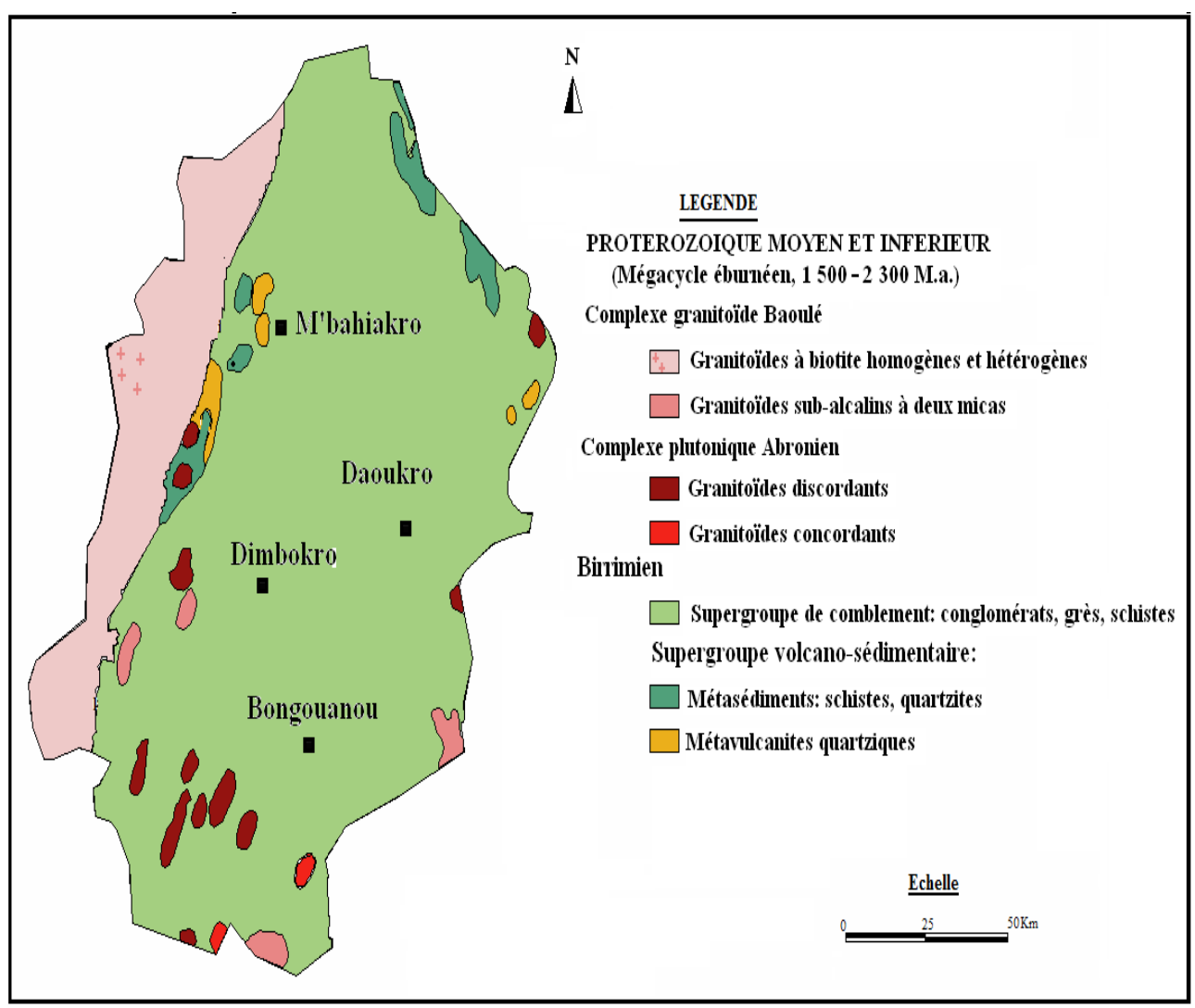

Figure 2: Carte géologique de la région du N’zi-Comoé (Centre-Est de la Côte d'Ivoire)

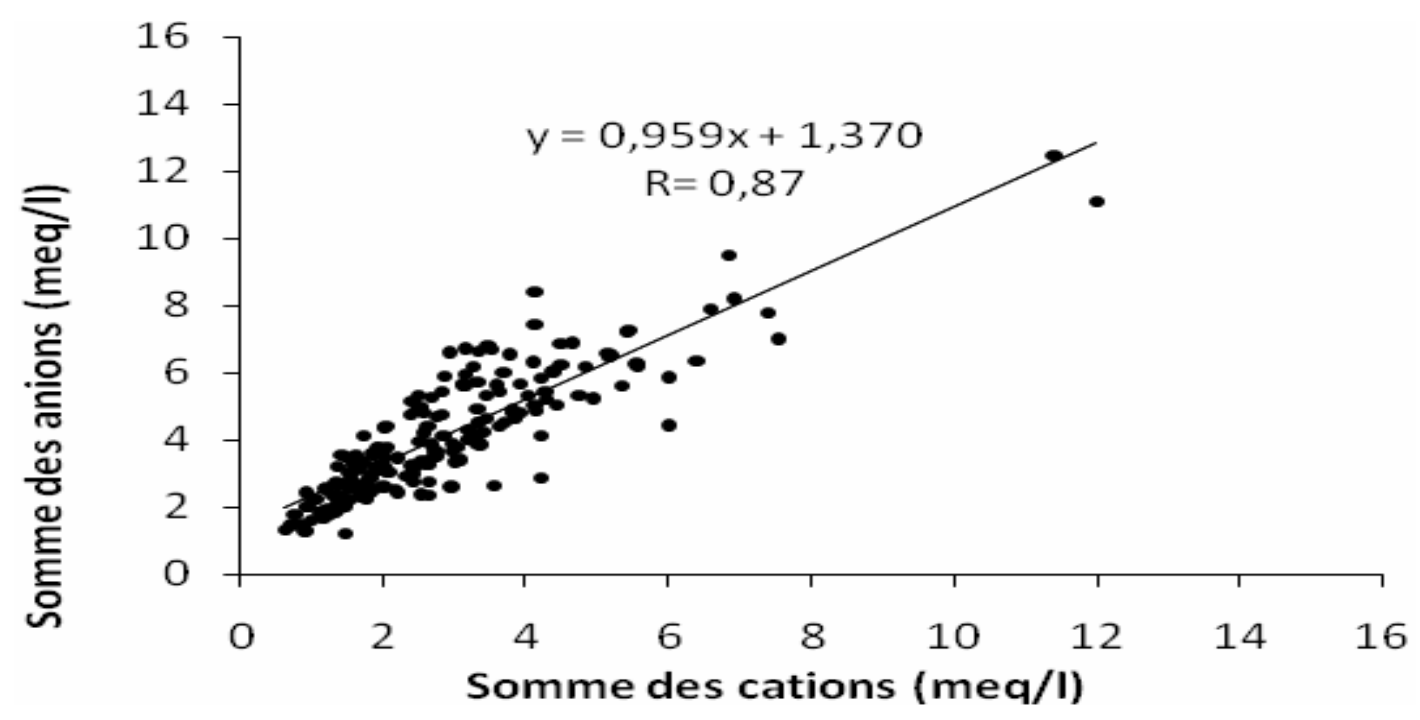

Figure 3 : Corrélation entre la somme des anions et la somme des cations 

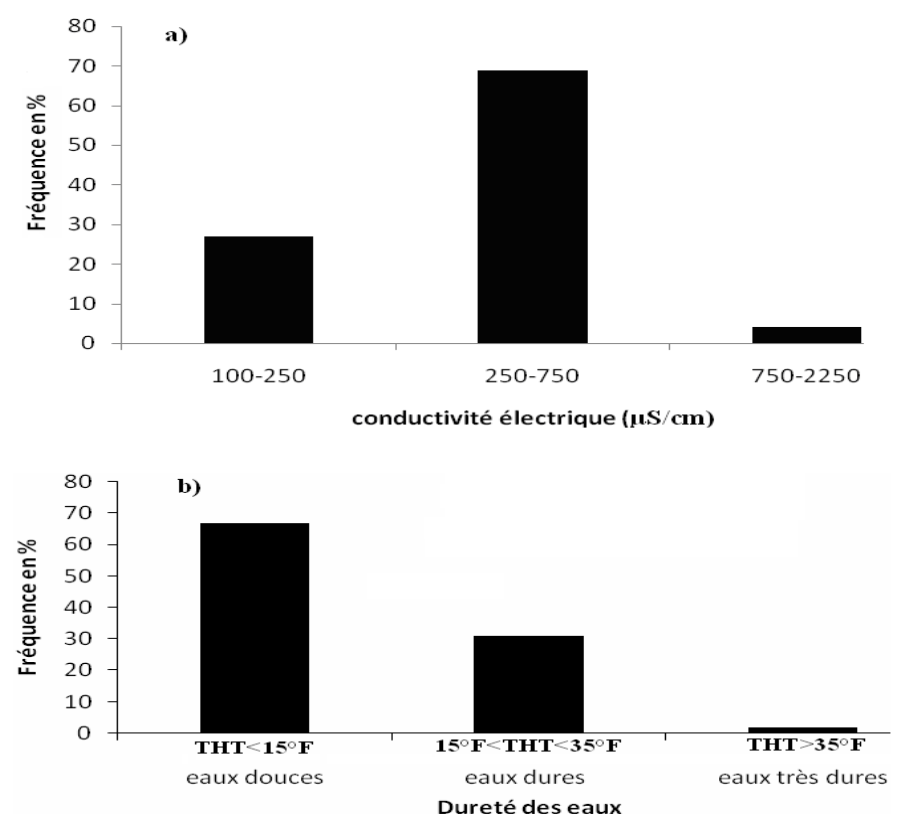

Figure 4: Histogrammes des fréquences de conductivité électrique (a) et dureté (b) des eaux souterraines de la région du N'zi-Comoé.

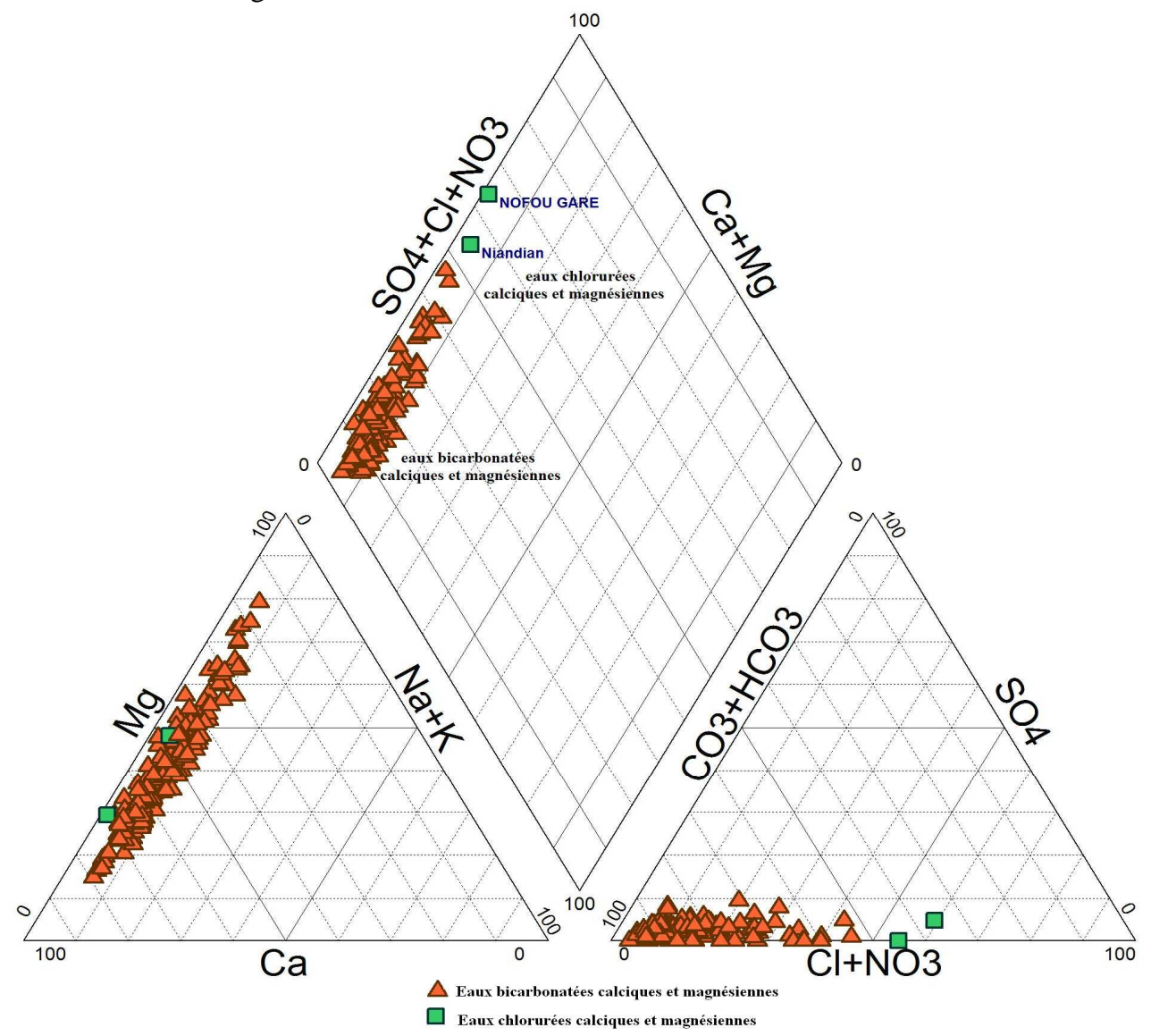

Figure 5: Classification des eaux souterraines du N'Zi-Comoé selon le diagramme de Piper. 


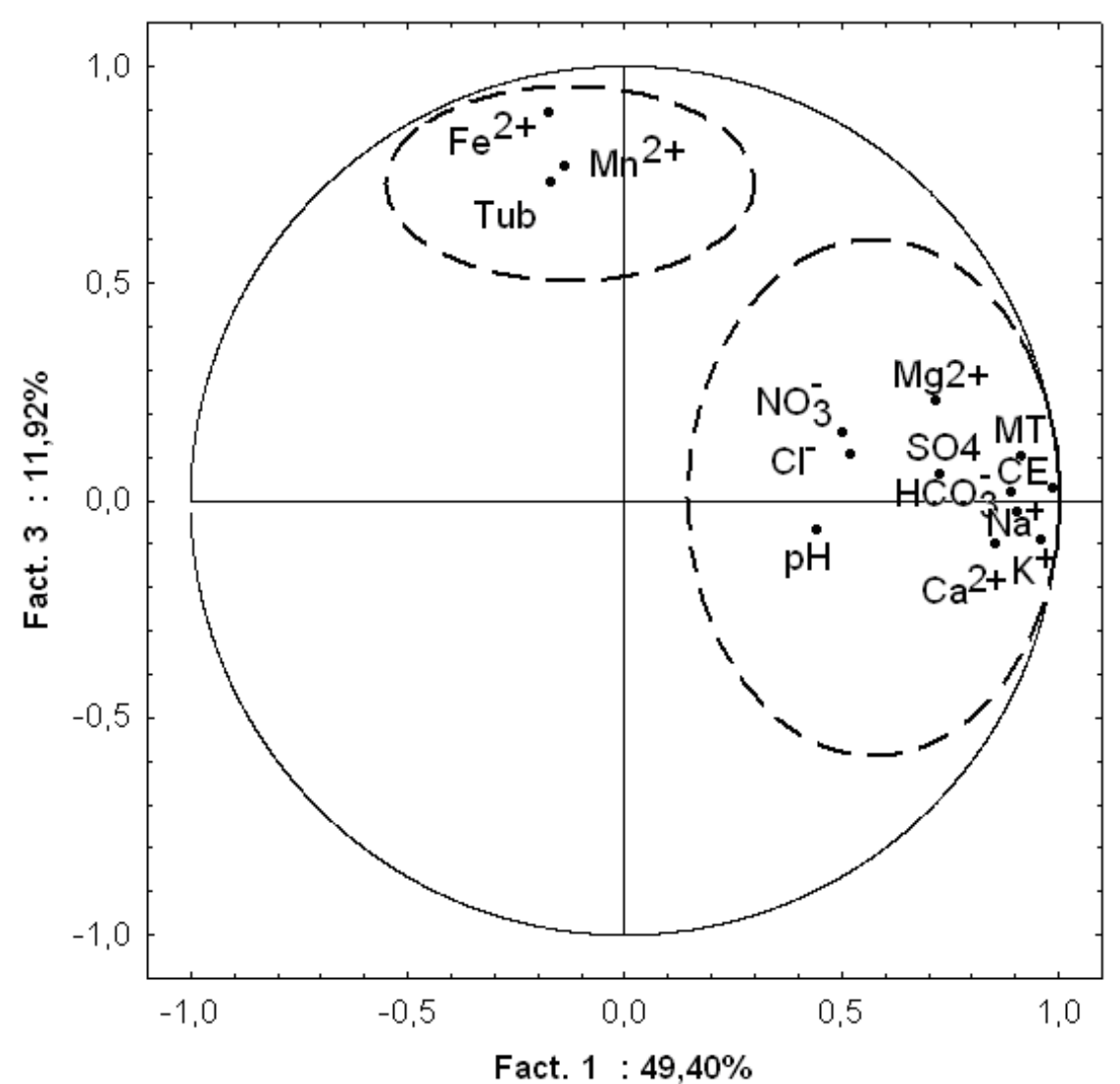

Figure 6: Analyse dans l'espace des variables (plan factoriel F1 - F3).

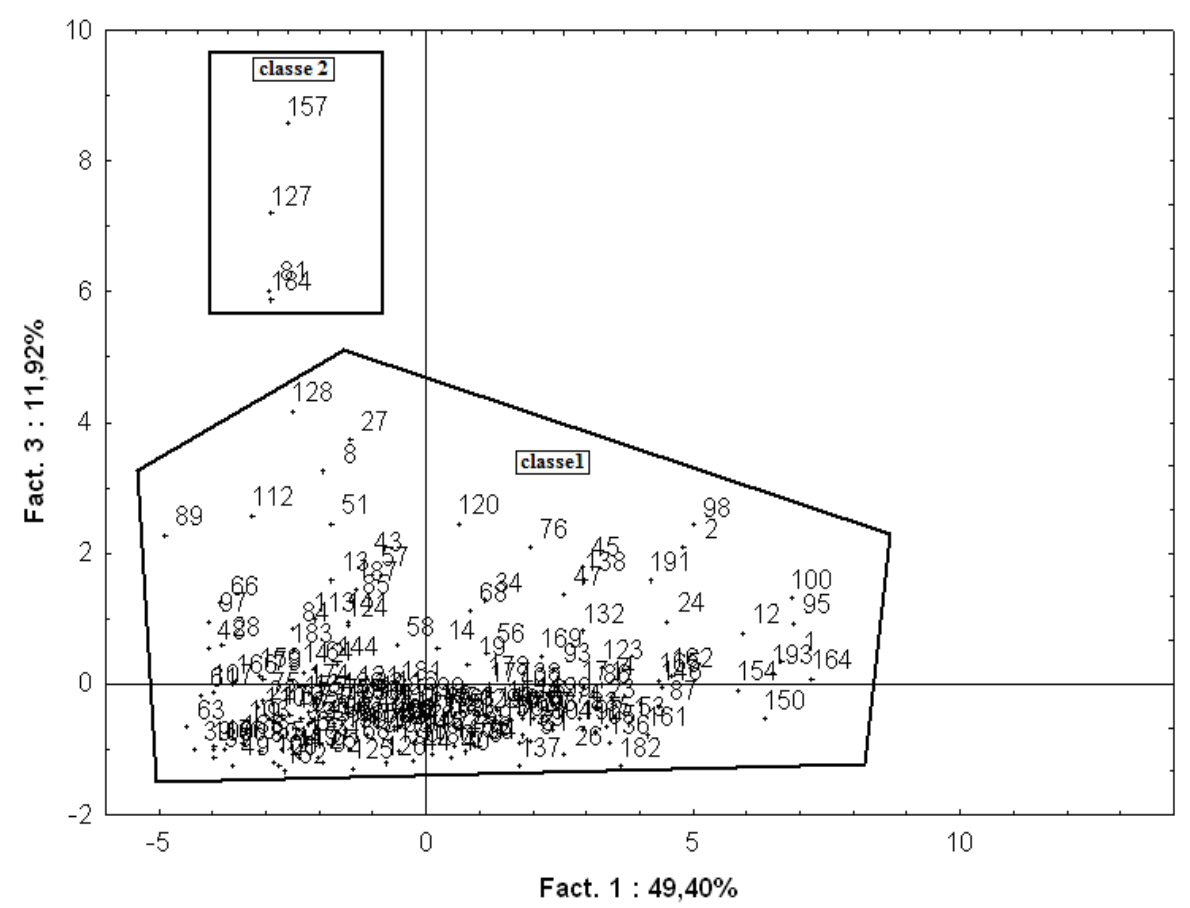

Figure 7: Analyse dans l'espace des unités statistiques (plan factoriel F1 - F3) 

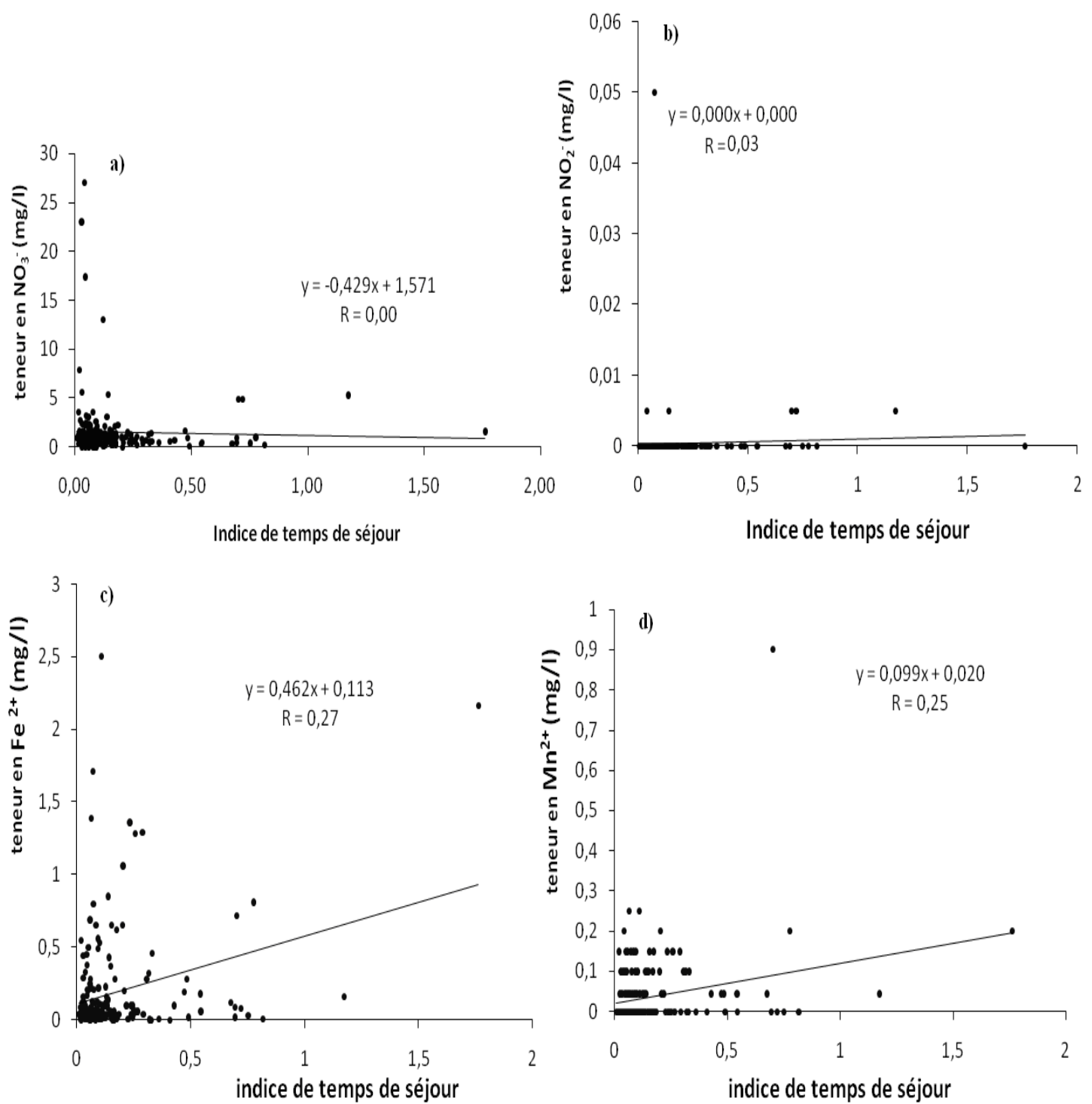

Figure 8 : Graphes de corrélations des éléments polluants en fonction des indices de temps de séjour.

\section{DISCUSSION}

Les températures des eaux souterraines étudiées varient autour de $27,18{ }^{\circ} \mathrm{C} \pm 1,37$. La norme étant fixée de 25 à $30^{\circ} \mathrm{C}$, ces eaux sont conformes à la recommandation de l'OMS. Le $\mathrm{pH}$ des eaux est majoritairement acide à neutre soit $86,5 \%$ des échantillons. D'après Lasm et al. (2008), cette acidité est principalement liée à la production de $\mathrm{CO}_{2}$ dans les couches superficielles du sol sous l'action des activités biologiques. La réaction d'hydratation du $\mathrm{CO}_{2}$ libère de l'acide carbonique qui attaque les roches (Goné et al., 2004 in Lasm et al., 2008). Les valeurs de la turbidité des eaux souterraines du N'zi-Comoé fluctuent autour de $0,79 \mathrm{NTU} \pm 0,37 \mathrm{NTU}$. Les eaux de la région du N'zi-Comoé contiennent donc peu de particules en suspension. D'une 
manière générale, les eaux sont en majorité moyennement minéralisées. Plusieurs travaux sont parvenus à cette conclusion en milieux de socle (Ahoussi, 2008 ; Lasm et al., 2008 ; Oga et al., 2009 ; Soro, 2010). Les forages à dureté indésirable (THT > $35^{\circ} \mathrm{F}$ ) sont caractérisés par des eaux difficilement moussantes, elles forment des flocons avec le savon. Ces eaux sont aussi caractérisées par un dépôt au fond des casseroles lorsqu'elles sont portées à ébullition (Tardat et al., 1984 in Lasm et al., 2008).

Du point de vue hydrofaciès, la quasitotalité des eaux des aquifères fissurés de la région du N'Zi-Comoé appartient au faciès bicarbonaté calcique et magnésien. C'est une caractéristique des eaux du socle, qui diffèrent des eaux souterraines du bassin sédimentaire de Côte d'Ivoire qui présentent majoritairement un faciès chloruré sodipotassique (Ahoussi, 2008). Cette quasihomogénéité des hydrofaciès de la zone d'étude est liée à sa faible hétérogénéité lithologique. En effet, la géologie du N'ZiComoé est largement dominée par les schistes ; environ $86,46 \%$ des eaux du N'ZiComoé sont contenues dans des aquifères schisteux et seulement environ $15,54 \%$ des aquifères sont granitiques ou gréseux.

La prédominance des bicarbonates dans les eaux de la région du N'zi-Comoé est une caractéristique des eaux souterraines des régions de socle de l'Afrique de l'Ouest en général et de la Côte d'Ivoire en particulier (Soro, 2002 ; Oga et al., 2009). L'abondance des ions bicarbonates dans ces eaux pourrait s'expliquer par l'hydrolyse des feldspaths que l'on rencontre en abondance dans les roches acides (Goné et al., 2004 in Lasm et al., 2008). Les cations dominants qui s'associent à ces

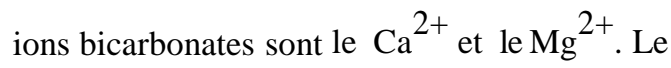
calcium provient des formations géologiques qui forment les aquifères, particulièrement les roches volcaniques basiques métamorphisées (métabasalte, métaandésite, métadolérite, métagabbro, amphibolite) (Faillat et blavoux, 1989). La minéralisation calcique des eaux du socle est sous l'influence de l'altération et de l'hydrolyse des minéraux alumino-silicates riches en $\mathrm{Ca}^{2+}$. La source du magnésium est liée à la décomposition des minéraux ferromagnésiens tels que la biotite et l'amphibole contenus dans les roches vertes de la région. La prédominance des ions $\mathrm{Na}^{+}$sur les ions $\mathrm{K}^{+}$dans les eaux souterraines de la région du N'zi-Comoé peut s'expliquer d'une part par la grande stabilité de la muscovite et des feldspaths potassiques et d'autre part par l'adsorption des ions potassium par les plantes (Oga et al., 2009). Les ions $\mathrm{K}^{+}$peuvent également être adsorbés par les minéraux néoformés (Soro, 1987 in Oga et al., 2009). La minéralisation des eaux souterraines du N'zi-Comoé situées dans les terrains schisteux est plus élevée que celle sur les terrains granitiques. Cette conclusion corrobore les résultats des travaux de Oga et al. (2009) dans la région de Tiassalé (Côte d'Ivoire), située sur le socle.

Les résultats de l'ACPN montrent que les paramètres chimiques influençant la minéralisation des eaux de la région du N'ziComoé sont, par ordre d'influence croissante, le potassium, le sodium, le bicarbonate, le magnésium et le calcium. En effet, selon Rabemanana (2002), la minéralisation due à l'interaction eau-roche est acquise par l'hydrolyse des silicates sous la condition de milieu ouvert. Les eaux à fortes teneurs en fer et en manganèse ont des teneurs en composés azotés (nitrates, nitrites) faibles. Rabemanana (2002) est aussi parvenu à cette conclusion au cours de ses travaux.

Les teneurs en nitrates sont toutes en dessous de la norme fixée par l'OMS (50 $\mathrm{mg} / \mathrm{l})$. Cependant, $42 \%$ des points d'eau ont des teneurs en nitrate supérieures à $1 \mathrm{mg} / \mathrm{l}$ dont $6,17 \%$ ont des teneurs supérieures à $10 \mathrm{mg} / \mathrm{l}$. Selon Landreau et Roux (1985), à l'état naturel, la teneur en nitrates dans la nappe souterraine varie de 0,1 à $1 \mathrm{mg}$ par litre d'eau. Ce fort taux de nitrate de teneurs supérieures à $1 \mathrm{mg} / \mathrm{l}$ traduit donc un apport de nitrate. D'où vient donc cet apport? 
Les nitrites sont quasi inexistants dans les eaux de la région du N'zi-Comoé. Néanmoins, 3,11\% des points d'eau ont des teneurs non nuls $(0,005-0,05 \mathrm{mg} / \mathrm{l})$, qui sont en dessous de la norme OMS $(0,1 \mathrm{mg} / \mathrm{l})$. Cependant, cette présence de nitrite pourrait susciter des inquiétudes. Les différents points d'eau affectés par la présence de nitrite sont localisés dans le département de Bongouanou. Ce département concentre $38 \%$ de la population agricole de la région du N'ziComoé et le tiers du cheptel de la région.

La présence de fortes teneurs en nitrates dans les eaux souterraines de la région du N'zi-Comoé (ancienne boucle du cacao en Côte d'Ivoire) a été déjà constatée par certains auteurs tels que Faillat et Blavoux (1989), Faillat (1990), etc. Ces taux de nitrate aux caractères polluants, semblent suggérer des apports ponctuels ou sur des superficies restreintes, d'origines naturels ou anthropiques. En effet, les roches du socle métamorphique sont peu favorables à la présence des minéraux nitratés. Seuls les micas (biotites, etc.) sont susceptibles de contenir des quantités notables d'azote sous forme de $\mathrm{NH}_{4}{ }^{+}$(Moine et al., 1994 ; Schroeder et al., 1994 ; Holloway et al., 1999 cités par Rabemanana, 2002). Par exemple, la teneur en azote total dans la biote de certains schistes peut varier de 253 à $1321 \quad \mathrm{mg} \mathrm{NKg}^{-1}$ (Holloway, 1998 in Rabemanana, 2002). Ce qui est très faible. Il semble donc que le taux élevé de composés azotés (nitrates, nitrites) serait lié à une origine anthropique. Selon les travaux de Faillat et Blavoux (1989), à partir d'une série de 230 analyses hydrochimiques des éléments majeurs, il ressort que les facteurs de contrôle du chimisme des eaux des nappes de fissures sont la lithologie, la végétation et l'action anthropique. Des travaux plus approfondis de Faillat (1990), ont montré que de fortes teneurs en nitrates, jusqu'à 200 mg $\mathrm{l}^{-1}$, ont été observées dans les nappes de fissures sous des altérites épaisses, dans la zone tropicale humide à pluies annuelles supérieures à $1000 \mathrm{~mm}$. Selon le même auteur, le lessivage des sols et des débris végétaux en cours de décomposition, après déforestation explique en partie les taux élevés de nitrate. Ainsi, les nitrates des eaux souterraines auraient pour origine les réactions d'oxydoréduction de matières organiques liées aux activités humaines ou végétales. En effet, l'azote représente $3,5 \%$ en poids d'une plante banale vivante et $1,5 \%$ de sa matière sèche (Faillat et Rambaud, 1991 in Soro, 2010). Faillat et Rambaud (1991) cités par Soro (2010) estiment à 1500 tonnes par hectare de matière végétale en forêt intertropicale humide, soit un maximum de 50 tonnes par hectare d'azote disponible. Cependant, même s'il est difficile d'apprécier la part d'azote qui, d'une part, sera nitrifiée, et d'autre part, parviendra aux nappes sous formes de $\mathrm{NO}_{3}{ }^{-}$, compte tenu des phénomènes de ruissellement, d'infiltration, de fixation ou de dénitrification dans les sols, il est certain qu'une partie non négligeable arrive aux eaux souterraines, et contribue aux teneurs anormalement élevées. La zone d'étude étant suffisamment fracturée (Daouda, 1998; Kadio et al., 2008), la recharge des aquifères de socle est contrôlée par le réseau fracturé et les cours d'eau qui empruntent des accidents majeurs. Les fissures et fractures associées à ces aquifères assurent le transit souterrain des nitrates par lessivage jusqu'à contaminer la nappe. Aussi, l'utilisation même faible d'engrais suite à l'appauvrissement des sols issu de la déforestation dans la région du N'zi-Comoé, ancienne boucle du cacao, pourrait justifier en partie l'apport polluant des composés azotés (nitrates, nitrites) dans les eaux étudiées. Plusieurs études réalisées en Afrique ont contribué à montrer que les nitrates ont une origine liée aux apports de fertilisants, enrichissant les eaux souterraines par lessivage des sols agricoles (Dakouré, 2003 ; Ahoussi, 2008 ). Cette thèse est aussi partagée par Bricha et al. (2007), pour qui, la pollution nitrique des eaux souterraines peut être due à l'utilisation des fertilisants azotés et à l'élevage. Selon les résultats des travaux de 
Soro (2010) dans la région des lacs (Côte d'Ivoire), les teneurs les plus élevées en nitrates sont localisées dans les zones rurales où l'activité principale est l'agriculture. Il y a de fortes raisons de s'attendre au cours des décennies à venir, à une augmentation du taux de nitrate dans les eaux souterraines de la région du N'zi-Comoé due à l'effet des activités anthropiques (déforestation, usage d'engrais).

Les fortes teneurs en fer et en manganèse enregistrées dans certaines localités constituent un important souci. En effet, ces deux éléments confèrent à l'eau un goût métallique désagréable, un aspect et une couleur (rouge brun et marron noir respectivement pour le fer et pour le manganèse) qui amènent les populations rurales à se tourner vers d'autres sources d'approvisionnement dont les qualités bactériologiques et parasitologiques sont douteuses. Ce qui peut avoir des conséquences graves sur la santé des populations. Par ailleurs, une telle eau laisse des taches sur la lingerie. Selon la concentration, il peut se produire des précipitations sur les parois des canalisations diminuant considérablement leur diamètre utile, provoquant ainsi un colmatage au niveau de ces canalisations (Lasm et al., 2008). Un développement de bactéries ferrugineuses augmentant ainsi la corrosion pourrait être observé dans les forages, les rendant vulnérables à d'autres sources de pollution qui dégraderaient considérablement la qualité de ces eaux (Oga et al., 2009). En outre, le fer peut être en solution vraie, à l'état colloïdal, plus ou moins combiné à la matière organique, sous forme de complexes organiques ou minéraux ou sous forme de particules en suspension. Au contact de l'air, l'ion ferreux $\mathrm{Fe}^{2+}$ (soluble) est oxydé en ion ferrique $\mathrm{Fe}^{3+}$ (insoluble). Ainsi, l'oxydation du fer contenu dans l'eau confère à celle-ci sa turbidité et sa couleur rouge parfois observée dans les eaux souterraines (Lasm et al., 2008). Le manganèse est l'élément chimique qui accompagne généralement le fer dans les roches. Il provient en général des cuirasses rencontrées dans la région. Le fer et le manganèse semblent provenir de l'altération des roches de surface (Lasm et al., 2008). En effet, l'essentiel des acides secrétés par les micro-organismes (algobactéries et microbes silicophiles) favorise la dissociation des minéraux des roches notamment la silice, les silicates (d'alumine, de potassium, de fer et de magnésium) et la matière organique pour libérer les ions tels que le $\mathrm{Fe}^{2+}$. A la suite d'un phénomène de pluviolessivage, ces éléments vont être transportés et entraînés vers les aquifères des eaux souterraines. Ces roches sont riches en minéraux ferromagnésiens notamment en pyroxènes, amphiboles et en biotites. L'altération de celles-ci libère entre autres les $\mathrm{Fe}^{2+}$ qui sont par la suite entraînés vers les eaux souterraines. La région étant abondamment arrosée, le pluviolessivage des couches superficielles entraîne un départ important de plusieurs cations dont le $\mathrm{Mn}^{2+}$ vers les nappes (Biémi, 1992). Selon Soro (2010), le manganèse est le principal constituant des cuirasses manganésifères. Il y est présent sous forme d'oxyde manganifère. La réaction entre ces oxydes en milieu anaérobie dans des eaux moyennement acide produit des ions manganèses $\left(\mathrm{Mn}^{2+}\right)$.

Les faibles corrélations obtenues entre les indicateurs de temps de séjour et les métaux (le fer et le manganèse) confirment que l'oxydo-réduction et le lessivage des silicates ferromagnésiens semblent donc être les sources probables du fer et du manganèse dans les eaux de la région du N'zi-Comoé. Ces résultats confirment ceux obtenus par Soro (2010) dans la région des lacs (Côte d'Ivoire).

\section{Conclusion}

La caractérisation hydrogéochimique des eaux des aquifères fissurés de la région du N'zi-Comoé (Centre-Est) de la Côte d'Ivoire à partir de la combinaison des méthodes 
hydrochimiques (diagramme de piper, indicateur de temps de séjour) et d'analyses statistiques univariées, bivariées et multivariées (ACPN) a conduit à la mise en évidence des différentes caractéristiques des eaux souterraines de la région et leur aptitude à la consommation humaine. Les eaux souterraines ont des températures variant entre $24,4{ }^{\circ} \mathrm{C}$ et $31,6{ }^{\circ} \mathrm{C}$ et un $\mathrm{pH}$ généralement acide $(6,58)$. Les eaux souterraines du N'ziComoé contiennent très peu de particules en suspension ce qui se traduit par des valeurs de turbidité qui restent faibles dans l'ensemble (0,33 NTU à 3,49 NTU). Les points d'eau ont généralement des conductivités électriques comprises entre $250 \mu \mathrm{s} / \mathrm{cm}$ et $750 \mu \mathrm{s} / \mathrm{cm}$ (69\%), ce qui correspond aux eaux moyennement minéralisées. Les eaux étudiées sont généralement douces (près de 70\%). Du point de vue hydrofaciès, les eaux des aquifères fissurés du N'zi-Comoé sont en général du faciès bicarbonaté calcique et magnésien (99\%). Ce faciès est typique des eaux des aquifères de socle de Côte d'Ivoire. Deux phénomènes hydrochimiques majeurs sont observables dans la zone d'étude. Il s'agit du phénomène de minéralisation couplé au temps de séjour qui est à l'origine des teneurs en calcium et en magnésium, et du phénomène d'oxydo-réduction lié au fer et au manganèse et responsable de la turbidité. L'étude de la potabilité révèle que les eaux du N'zi-Comoé sont physico-chimiquement conformes dans l'ensemble et ne présentent pas de danger majeur pour la consommation humaine. Cependant, les valeurs de nitrates obtenues dans les eaux, bien qu'en dessous de la norme OMS, traduisent un début de pollution localisée et d'origine anthropique. De plus, les fortes teneurs relativement élevées en fer et en manganèse enregistrées dans certaines localités, constituent un souci important.

\section{REMERCIEMENTS}

Les auteurs de cet article remercient les instructeurs dont les critiques et les suggestions ont permis d'améliorer le présent article. Ils remercient également la Direction de l'Hydraulique Humaine (DHH) pour leur avoir fourni les données d'analyses physicochimiques utilisées dans cette étude.

\section{REFERENCES}

Ahoussi KE. 2008. Evaluation quantitative et qualitative des ressources en eau dans le Sud de la Côte d'Ivoire. Application de l'hydrochimie et des isotopes de l'environnement à l'étude des aquifères continus et discontinus de la région d'Abidjan-Agboville. Thèse de Doctorat de l'Université de Cocody-Abidjan, Côte d'Ivoire, p. 270.

Alami II, Zeraouli M, Addou M, Mokhtari A, Soulaymani A. 2007. Évaluation de la pollution nitrique de la nappe phréatique de la zone côtière du Gharb (M'nasra) au Maroc entre 1993 et 2003. Afrique Science, 3(3): 378-390.

Alayat H, Lamouroux C. 2007. Caractérisation physico-chimique des eaux thermo-minérales des monts de la Cheffia (extrême Nord-Est algérien). La Presse Thermale et Climatique, 144: 191199.

Anonyme. 2009. Etats généraux de l'eau potable en Côte d'Ivoire. Ministère des infrastructures économiques, Office National de l'Eau Potable (ONEP), p.6.

Avenard JM, Eldin M, Girard G, Sircoulon J, Touchebeuf P, Guillaumet JL, Adjanohoun E, Perraud A. 1973. Une monographie physique de la Côted'Ivoire. Annales de Géographie, 82(451): 369 - 372

Bahir M, Carreira P, Da Silva MO, Fernandes P. 2008. Caractérisation hydrodynamique, hydrochimique et isotopique du système aquifère de Kourimat (Bassin d'Essaouira, Maroc). Estudios Geológicos, 64(1): 61-73.

Biémi J. 1992. Contribution à l'étude géologique, hydrogéologique et par télédétection des bassins versants 
subsahariens du socle précambrien d'Afrique de l'Ouest : hydrostructurale, hydrodynamique, hydrochimie et isotopique des aquifères discontinus de sillons et des aires granitiques de la Haute Marahoué (Côte d'Ivoire). Thèse de Doctorat d'Etat, Université de CocodyAbidjan, Côte d'Ivoire, p.480.

Bois PH, Obled CH, Zin I. 2007. Introduction au traitement de données en hydrologie. Fascicule, Institut National Polytechnique de Grenoble, $7^{\text {ème }}$ édition revue et complétée, p. 265.

Bonvallot J, Boulangé B. 1970. Note sur le relief et son évolution dans la région de Bongouanou (Côte d'Ivoire). Cahier de l'ORSTOM, Série Géologie, 2(2): 171183.

Boulangé B, Delvigne J. 1973. Descriptions morphoscopiques, géochimiques et minéralogiques des faciès cuirassés des principaux niveaux géomorphologiques de cote d'ivoire (1). Cahier de l'ORSTOM, Série Géologie, 5(1): 59-81.

Bricha S, Ounine K, Oulkheir S, El Haloui N, Attarassi B. 2007. Etude de la qualité physicochimique et bactériologique de la nappe phréatique M'nasra (Maroc). Afrique Science, 3(3): 391-404.

Brou YT. 2005. Climat, mutations socioéconomiques et paysages en Côte d'Ivoire. Mémoire de synthèse des activités scientifiques présenté en vue de l'obtention de 1'Habilitation à Diriger des Recherches, Université des Sciences et Technologies de Lille, France, p.226.

Chemseddine F, Abderrahmane B, Abdelkader R, Elias S. 2009. Caractérisation hydrogéochimique des eaux souterraines du complexe aquifère Morsott-Laouinet (Région Nord de Tébessa, Sud-Est algérien). Afrique Science, 5(2): 217-231.

Collectif. 1984. Synthèse des Connaissances sur l'Hydrologie du Socle Cristallin et Cristallophyllien et du Sédimentaire Ancien de l'Afrique de l'Ouest (1ère édn).
C.E.F.I.G.R.E : Sophia Antipolis, France ; 121.

Dakouré D. 2003. Etude hydrogéologique et géochimique de la bordure sud-est du bassin sédimentaire de Taoudéni (Burkina Faso - Mali) - essai de modélisation. Thèse de Doctorat, Université Paris VI, France, p. 255.

Daouda YB. 1998. Lithostratigraphie et pétrographie des formations birimiennes de Toumodi-Fêtêkro (Côte d'Ivoire). Implication pour l'évolution crustale du paléoprotérozoique du craton ouestafricain. Thèse de Doctorat de l'Université d'Orléans, France, p.190.

El Bouqdaoui K, Achib M, Blaghen M, Kholtei S. 2009. Modélisation de la pollution par les nitrates de la nappe de Berrechid, au Maroc. Afrique Science, 5(1): 99-113.

Faillat JP, Blavoux B. 1989. Caractères hydrochimiques des nappes des roches endogènes fissurées en zone tropicale humide : l'exemple de la Côte d'Ivoire. $J$. Afri. Earth. Sc., 9(1): 31- 40.

Faillat JP. 1990. Origine des nitrates dans les nappes de fissures de la zone tropicale humide. Exemple de la Côte d'Ivoire. Journal of Hydrol., 113: 231-264.

Géomines L. 1982a. Inventaire hydrogéologique appliqué à l'hydraulique villageoise. Ministère des Travaux Publics et des Transports, Direction Centrale de l'Hydraulique, République de Côte d'Ivoire, carte de M'bahiakro, Cahier $\mathrm{n}^{\circ} 19, \mathrm{p} .20$.

Géomines L. 1982c. Inventaire hydrogéologique appliqué à l'hydraulique villageoise. Ministère des Travaux Publics et des Transports, Direction Centrale de l'Hydraulique, République de Côte d'Ivoire, carte de Dimbokro, Cahier $\mathrm{n}^{\circ} 25, \mathrm{p} .20$.

INS. 2001. Données socio-démographiques et économiques des localités, résultats définitifs par localités, région du N'ziComoé. Recensement Général de la 
Population et de l'Habitat (RGPH) 1998. INS.

Kadio NH, Saley MB, N'dri BE, Ouattara A, Biemi J. 2008. Contribution à l'interprétation des linéaments par l'exploitation des Pseudo images, de l'hydrographie en région tropicale humide: cas du N'zi-Comoé (Centre de la Côte d'Ivoire). European Journal of Scientific Research, 24(1): 74-93.

Kouassi AM. 2007. Caractérisation d'une modification éventuelle de la relation pluie-débit et ses impacts sur les ressources en eau en Afrique de l'Ouest : cas du bassin versant du N'zi (Bandama) en Côte d'Ivoire. Thèse de Doctorat, Université de Cocody-Abidjan, Côte d'Ivoire, p. 210.

Kouzana L, Ben Mammou A, Gaaloul N. 2007. Intrusion marine et salinisation des eaux d'une nappe phréatique côtière (Korba, Cap-Bon, Tunisie). Geo-EcoTrop., 31: 57-70.

Lalbat F. 2006. Fonctionnement hydrodynamique de l'aquifère du Miocène du bassin de Carpentras (Vaucluse, France). Thèse de Doctorat, Université d'Avignon et des pays de Vaucluse, France, p. 234.

Laborde JP. 2000. Eléments d'hydrologie de surface. Fascicule de cours, Université de Nice, France, p. 191.

Lallahem S. 2002. Structure et modélisation hydrodynamique des eaux souterraines : application à l'aquifère crayeux de la bordure nord du bassin de paris. Thèse de Doctorat, Université de Lille, France, p. 243.

Landreau A, Roux JC. 1985. Les Nitrates dans les Eaux Souterraines. Exemples de Répartition et d'Evolution des Teneurs dans quelques Aquifères Français. BRGM, Edition CEDEX : Orléans ; 55.

Lasm T, Yao KT, Oga MS, Kouame KF, Jourda P, Kouadio KE, Baka D. 2008. Analysis of the physico-chemical characteristics of groundwater in proterozoic land region of the Tiassale area (Southern Côte d'Ivoire). European Journal of Scientific Research, 20(3): 526-543.

Matini L, Moutou JM, Kongo-Mantono MS. 2009. Evaluation hydro-chimique des eaux souterraines en milieu urbain au Sud-Ouest de Brazzaville, Congo. Afrique Science, 5(1): 82-98.

Mohammad RAA, Hamed AEN. 2004. Hydrochemical facies of groundwater in the Gaza Strip, Palestine. Journal des Sciences Hydrologiques, 49(3): 359-371.

Oga MS, Lasm T, Yao KT, Soro N, Saley MB, Kouassi D, Gnamba F. 2009. Caractérisation chimique des eaux des aquifères de Fracture: cas de la région de Tiassalé en Côte D'ivoire. European Journal of Scientific Research, 31(1): 7287.

Okaingni JC, Kouamé KF, Martin A. 2009. Cartographie des cuirasses dans les formations volcano-sédimentaires de la zone d'Anikro-Kadiokro (Côte d'Ivoire) à l'aide de la théorie des fonctions de croyance. Revue Télédétection, 9(1): 1932.

OMS. 2006. Normes de l'OMS sur l'eau potable.

Rabemanana V. 2002. Origine et caractérisation de la salinité des eaux dans les aquifères de socle. Thèse de Doctorat de l'Université Paris VI, France, p.182.

Soro N. 2002. Hydrochimie et géochimie isotopique des eaux souterraines du degré carré de Grand-Lahou et ses environs (Sud-Ouest de la Côte d'Ivoire). Implication hydrologique et hydrogéologique. Thèse de Doctorat ès sciences naturelles, Université de Cocody-Abidjan, Côte d'Ivoire, p. 272.

Soro G. 2010. Évaluation quantitative et qualitative des ressources en eaux souterraines dans la région des lacs (centre de la côte d'ivoire) : Hydrogéologie et hydrochimie des 
aquifères discontinus du district de Yamoussoukro et du département de Tiebissou. Thèse de Doctorat, Université de Cocody-Abidjan, Côte d'Ivoire, 257 p.

Tabouche N, Achour S. 2004. Etude de la qualité des eaux souterraines de la région orientale du Sahara septentrional algérien. Larhyss Journal, 3: 99-113.

Touaïbia B, Inegliz S, Ould AA. 2006. Couplage d'une analyse en composantes principales et d'une approche géostatistique pour l'élaboration de cartes pluviométriques du Centre de l'Algérie du Nord ». Revue des Sciences de l'Eau, 19(3): 213-219.

Yermani M, Zouari K, Michelot JL, Mamou A, Moumni L. 2003. Approche géochimique du fonctionnement de la nappe profonde de Gafsa Nord (Tunisie centrale). Journal des Sciences Hydrologiques, 48(1): 95-108.

Yoboué KE, Yao-Kouamé A, Alui KA. 2010. Evolution Pédogéochimique et minéralogique au cours de l'altération des formations $\mathrm{du}$ complexe volcanosédimentaire d'Anikro et de Kahankro (Toumodi) dans le Centre-Sud de la Côte d'Ivoire. European Journal of Scientific Research, 40(1): 60 -72.

Zourhi L, Carlier E. 2002. Caractérisation hydrochimique d'une nappe côtière, Maroc. Journal of Environment Hydrology, 9(4): 1-7. 